\title{
Spleno-renal anastomotic bypass revascularization for worsening renal failure and uncontrolled hypertension in bilateral calcific atherosclerotic renal artery stenosis
}

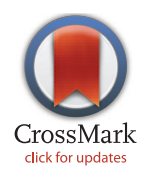

\author{
Macaulay Amechi Chukwukadibia Onuigbo* ${ }^{(\mathbb{D}}$, Marie Engesser, Sree Susarla \\ Division of Nephrology, Department of Medicine, The Robert Larner, M.D. College of Medicine, University of Vermont, Burlington, VT. \\ UHC Campus, 1 South Prospect Street, Burlington, VT 05401, USA
}

\section{A R T I C L E I N F O}

Article Type:

Case Report

\section{Article History:}

Received: 12 April 2019

Accepted: 2 July 2019

Published online: 27 July 2019

\section{Keywords:}

Hypertension

Renal artery stenosis

Renal failure

Renal revascularization

Spleno-renal bypass

Chronic kidney disease

\begin{abstract}
A B S T R A C T
Following the recent publications of the STAR-study, the ASTRAL trial, the HERCULES trial and the CORAL trial on renal revascularization versus medical therapy in the management of atherosclerotic renovascular disease, there has been a near paradigm shift implying the nonutility of revascularization as a useful and necessary therapeutic option. Our recent experience with a patient who underwent an anastomotic bypass revascularization for worsening renal failure and uncontrolled hypertension in bilateral calcific atherosclerotic renal artery stenosis in Burlington, VT rekindled this debate. We posit that in appropriately selected patients, patients with acutely worsening renal failure, uncontrolled hypertension and/or symptomatic pulmonary edema, there is indeed a place for revascularization therapy, especially in the light of improved and safer surgical and anesthesiology techniques. It must be correctly acknowledged that the above well popularized randomized trials recruited mostly patients with otherwise stable chronic kidney disease at the time of enrollment. Similarly, only $12 \%$ of the patients in both arms of the ASTRAL trial demonstrated rapidly worsening renal failure prior to enrollment
\end{abstract}

Implication for health policy/practice/research/medical education:

There continues an ongoing debate as to the appropriateness and/or utility of mechanical revascularization procedures in the management of atherosclerotic renovascular disease. In the light of more recent trails including the STAR-study, the ASTRAL trial, the HERCULES trial and the CORAL trial, the general consensus has gravitated towards the opinion that there is hardly any indication for such revascularization procedures versus medical therapy alone. Our recent experience with a female patient with symptomatic and progressive atherosclerotic renovascular disease who responded promptly to spleno-renal anastomosis has rekindled this controversy. We posit that in appropriately selected patients with a priori worsening renal failure, uncontrolled or accelerated hypertension and/or pulmonary edema, there is indeed a place for the mechanical therapeutic restoration of renal blood flow.

Please cite this paper as: Onuigbo MAC, Engesser M, Susarla S. Spleno-renal anastomotic bypass revascularization for worsening renal failure and uncontrolled hypertension in bilateral calcific atherosclerotic renal artery stenosis. J Renal Inj Prev. 2019;8(4):253-256. DOI: 10.15171/jrip.2019.48.

\section{Introduction}

In light of the findings of recent trials including the STAR-study, the ASTRAL trial, the HERCULES trial and the CORAL trial, there remains a need to reevaluate revascularization versus medical therapy of atherosclerotic renovascular disease (1-5). Furthermore, especially in the light of improved and safer surgical and anesthesiology techniques, the role of revascularization in this context demands additional contemporary review (6-
10). Definitely, the appropriate patient selection for renal revascularization plays a pivotal role in determining the postoperative outcomes (6). Therapeutic revascularization can be helpful in appropriate circumstances where pharmaceutical intervention has failed and significant renovascular disease was contributing to treatmentresistant hypertension (10). Moreover, rapid deterioration in previously stable renal function or the new onset of uncontrollable hypertension herald potential renovascular 
disease and should prompt additional therapies, other than medical therapy alone (6-10). Our case report has enabled us to refocus attention to this debate.

\section{Case Report}

A 73-year old Caucasian woman with a past medical history including hypertension and postpartum deep vein thrombosis (DVT) (2007) with known heterozygous oneallele positive tests for both Factor V Leiden mutation and Prothrombin Gene PTG20210A mutation was evaluated for rising serum creatinine and worsening hypertension in 2018 (Figure 1). Five years earlier renal Doppler evaluation had demonstrated a $>60 \%$ left renal artery stenosis which was not further worked up since she then had stable kidney function and controlled hypertension. Indeed, her serum creatinine had remained stable (0.70-0.90 $\mathrm{mg} / \mathrm{dL}$ ) for more than 15 years, between 2000 and 2016 (Figure 1). However, in August 2018, her serum creatinine had risen to $2.14 \mathrm{mg} / \mathrm{dL}$ associated with hyperkalemia of $5.5 \mathrm{mmol} / \mathrm{L}$ (Figure 1). Her antihypertensive regimen consisted of amlodipine, $5 \mathrm{mg}$ daily and lisinopril, $40 \mathrm{mg}$ daily.

She had complained of fatigue present for several months, and was stressed out from the loss of the husband earlier in the year. She had lost about $14 \mathrm{~kg}$ weight. Except for occasional dizziness, she had no other specific symptoms. Blood pressure was 189/91, and pulse was a regular $87 /$ minute. She weighed $79.4 \mathrm{~kg}$, height was 161.3 $\mathrm{cm}$ and body mass index (BMI) was $30.51 \mathrm{~kg} / \mathrm{m}^{2}$. Physical examination was unremarkable except for an abdominal renal bruit. There was no edema, hepatomegaly nor raised jugular venous pressure. Chest was clear to auscultation. Dipstick urinalysis was negative. Lisinopril was promptly discontinued and replaced with Metoprolol XL $50 \mathrm{mg}$ daily. A new renal Doppler examination revealed $>60 \%$ stenosis in both renal arteries with normal resistive indices of 0.69 , respectively, with $75 \%-99 \%$ stenosis of the juxtarenal abdominal aorta which was heavily calcified

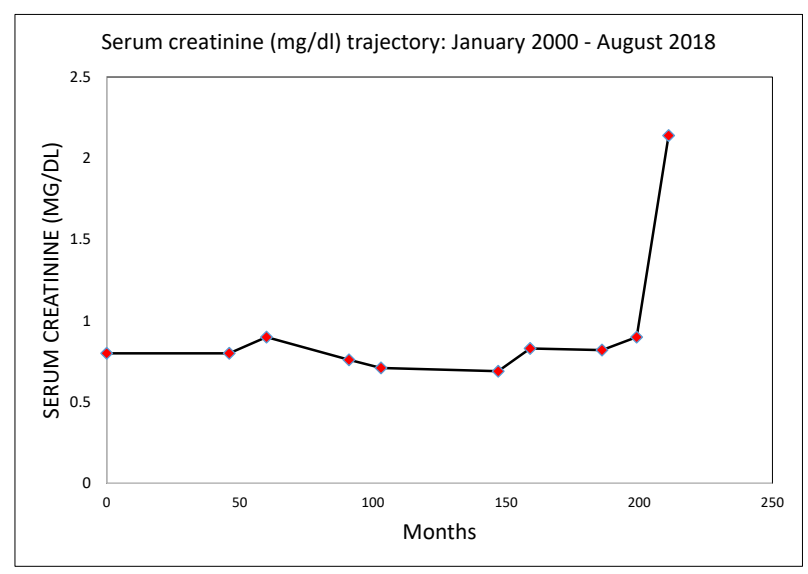

Figure 1. Stable serum creatinine from January 2000-July 2016, with an acute increase observed in August 2018.
(Figure 2). Both kidneys measured about $9.8 \mathrm{~cm}$ in length and showed no hydronephrosis. She was referred to Vascular Surgery for further evaluation for possible revascularization options.

A CT angiogram demonstrated severe calcified atherosclerosis with near complete occlusion of the pararenal abdominal aorta at the superior L 2 vertebral body level, a high-grade stenosis just above the iliac bifurcation, and moderate stenosis, nearly circumferential, within the remaining abdominal aorta (Figure 2). The celiac trunk, the superior mesenteric artery, and the left and right hepatic arteries were patent. The solitary left renal artery demonstrated near occlusive stenosis at its origin secondary to atherosclerotic plaque disease within the adjacent abdominal aorta. Two severely diminutive right renal arteries revealed severe ostial stenosis of the inferior right renal artery and severe near occlusive stenosis versus ostial occlusion of the superior renal artery (Figure 2). The inferior mesenteric artery was patent but severely attenuated and both common iliac arteries displayed severe ostial stenosis due to coarse calcified atherosclerotic plaques, but were otherwise patent with moderate stenosis. There was no evidence of pseudoaneurysm within the major arterial systems and the portal vein was patent (Figure 2). There was no hydronephrosis although the kidneys exhibited hypoenhancement and moderate cortical thinning, especially of the right cortical pole.

The reviewing vascular surgeon had noted that regarding the aortic disease, since the patient had experienced no history of intermittent claudication per se, and had no rest pain, the decision was made to only focus on renal preservation. Given the prior history of DVT and the presence of heterozygous prothrombotic genetic abnormalities, the plan was to use sequential compression devices preoperatively until she became ambulatory, to simultaneously start her on ultra-fractionated heparin (UFH) 5000 units SQ every 8 hours daily postoperatively when clinically stable (4-8 hours postoperatively), and to switch to oral apixaban $2.5 \mathrm{mg}$ twice daily for 30 days, at discharge, for anticoagulation prophylaxis. She was cleared for surgery following a nuclear cardiac stress test. Another preoperative mesenteric arterial system

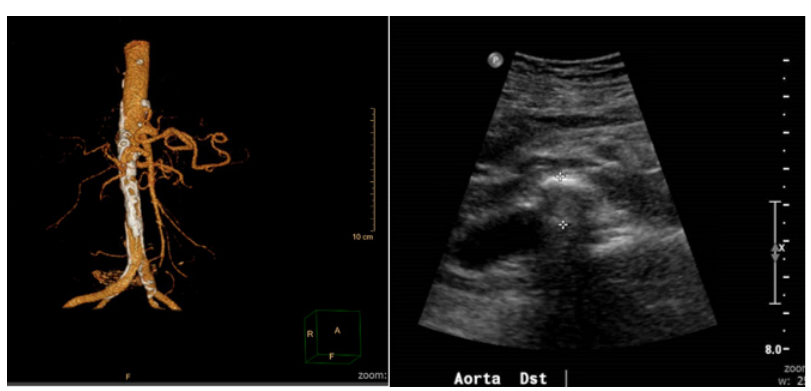

Figure 2. Composite showing CT Angiogram in $3 D$ and ultrasound Duplex showing calcific atherosclerotic stenosis of the aorta. 
ultrasound Doppler evaluation confirmed a widely patent celiac axis with no stenosis and the judgement was made by the vascular surgeon that the splenic artery would be a suitable inflow source for the planned bypass procedure. In the alternative, the right greater saphenous vein could serve as a suitable conduit for the planned bypass procedure, if otherwise indicated. She then underwent a left spleno-renal bypass, without complications under thoracic epidural anesthesia. Via a left subcostal incision, the redundant splenic artery was anastomosed in endto-end fashion to the left renal artery with a warm ischemia time of 17 minutes 20 seconds. The Doppler signal in the left kidney after the anastomosis was good. Postoperatively, she experienced no complications except that the systolic blood pressure was variable, and ranged from $140 \mathrm{~s}$ up to $180 \mathrm{~s} \mathrm{~mm} \mathrm{Hg.} \mathrm{After} \mathrm{the} \mathrm{surgery,} \mathrm{she} \mathrm{was}$ restarted on oral amlodipine $10 \mathrm{mg}$ daily together with as needed IV Labetalol dosed at $10 \mathrm{mg}$ every 4 hours, and IV hydralazine $10 \mathrm{mg}$ every 4 hours for systolic blood pressure $(\mathrm{SBP})>160 \mathrm{~mm} \mathrm{Hg}$. For persistent hypertension, on postoperative day 2, lisinopril was restarted at a dose of $40 \mathrm{mg}$ daily. Blood pressure was monitored very closely, and every effort was made to avoid precipitating hypotension.

The serum creatinine had improved to $1.68 \mathrm{mg} / \mathrm{dL}$, a CKD-EPI estimated glomerular filtration rate (eGFR) of $30 \mathrm{ml} / \mathrm{min} / 1.73 \mathrm{~m}^{2} \mathrm{BSA}, 2$ weeks before the surgery, about five months following the withdrawal of lisinopril (Figure 3). Following the left spleno-renal bypass procedure, the serum creatinine progressively fell and reached a value of $1.13 \mathrm{mg} / \mathrm{dL}(\mathrm{eGFR}=48)$ on post-operative day 3 (Figure 3). Nevertheless, the serum creatinine started to rise again thereafter and had risen up to $1.48 \mathrm{mg} / \mathrm{dL}(\mathrm{eGFR}=35)$ on post-operative day 5 , three days after restarting lisinopril (Figure 3). This rise in serum creatinine following the reinstitution of ACE inhibition was unacceptable and so the lisinopril was discontinued ${ }^{11}$ Lisinopril was replaced with carvedilol $12.5 \mathrm{mg}$ daily and minoxidil $2.5 \mathrm{mg}$ daily. She was discharged home with planned weekly basic metabolic profile testing and follow up. Blood pressure control on discharge from the hospital was good with SBP of 119-150 mm Hg and DBP of 51-81 mm Hg. The latest serum creatinine, about one month following the splenorenal bypass was $1.43 \mathrm{mg} / \mathrm{dL}(\mathrm{eGFR}=36)$.

\section{Discussion}

We had argued in 2015 that in patients with hemodynamically significant renal artery stenosis presenting with acutely worsening renal failure, that percutaneous transluminal renal angioplasty (PTRA) with stenting had the potential to reverse renal failure, improve blood pressure control, and mitigate flash pulmonary edema (5). Supporting evidence for this approach is well described (5-10). Likewise, when renal revascularization is thus indicated and PTRA and stenting is not possible,

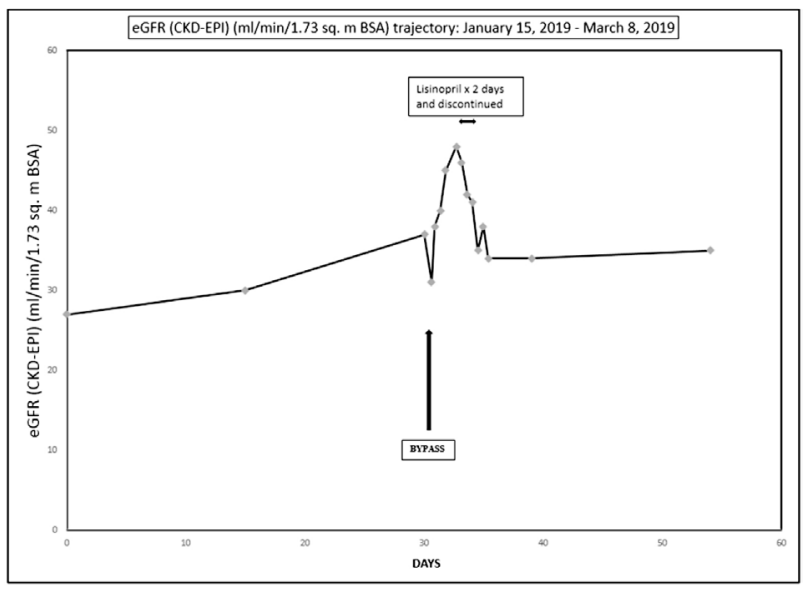

Figure 3. Estimated GFR trajectory one month before and one month following the spleno-renal bypass procedure.

then the need for surgical renal revascularization becomes inevitable (6-10). Unquestionably, appropriate patient selection for renal revascularization plays a pivotal role in determining the postoperative outcomes in such patients (6). Our patient demonstrated very rapid improvement in kidney function immediately following the successful spleno-renal end-to-end anastomosis with serum creatinine decreasing from $1.68 \mathrm{mg} / \mathrm{dL}$ to $1.13 \mathrm{mg} / \mathrm{dL}$ after only three days. (Figure 3 ). Some previous reports have demonstrated similar improvements in kidney function after renal revascularization $(6,9)$.

The reintroduction of angiotensin blockade in such patients following renal revascularization remains controversial. In our patient, the reintroduction of lisinopril on post-op day 2 for hypertension control led to a resurgence of worsening creatinine and its discontinuation (11). Other investigators have reported the successful use of angiotensin blockade following renal revascularization with no negative impact on subsequent renal recovery (10).

\section{Conclusion}

Finally, the reported negative or minimal impact of renal revascularization procedures versus medical therapy alone in the recent large trials, we posit, is the result of poor patient selection (1-4). Notably, the patients in the STAR, HERCULES, and CORAL trials had otherwise stable chronic kidney disease at the time of enrollment into these studies (1-3). Likewise, only $12 \%$ of the patients in both arms of the ASTRAL trial demonstrated rapidly worsening renal failure prior to enrollment (4). We would argue that in the appropriately selected patient with a priori worsening renal failure, uncontrolled or accelerated hypertension and/or pulmonary edema, there is a place for the mechanical therapeutic restoration of renal blood flow (5-10). This mechanical restoration of renal blood flow would either be PTRA or surgical revascularization 
as dictated by the extent of atherosclerotic renovascular disease (5-10).

\section{Authors' contribution}

MACO: Conception and design of the study, collection and assembly of data, data analysis and interpretation, literature review, final approval of the manuscript. ME: Collection and assembly of data, literature review, final approval of the manuscript. SS: Collection and assembly of data, literature review, final approval of the manuscript.

\section{Conflicts of interest}

The authors declare that they have no conflict of interest.

\section{Ethical considerations}

All procedures performed in studies involving human participants were in accordance with the ethical standards of the institutional and/or national research committee (include name of committee + reference number) and with the 1964 Helsinki declaration and its later amendments or comparable ethical standards. This case report was granted exemption from requiring ethics approval, following a review with the Chair of The Robert Larner, M.D. College of Medicine, University of Vermont Institutional Review Board (IRB). The exemption was granted as a matter of policy with single case reports in the institution with deidentification.

\section{Funding/Support}

None.

\section{References}

1. Bax L, Mali WP, Buskens E, Koomans HA, Beutler JJ, Braam $\mathrm{B}$, et al. The benefit of stent placement and blood pressure and lipid-lowering for the prevention of progression of renal dysfunction caused by Atherosclerotic ostial stenosis of the Renal artery. The STAR-study: rationale and study design. J Nephrol .2003;16:807-812.

2. Wheatley K, Ives N, Gray R, Kalra PA, Moss JG, Baigent $\mathrm{C}$, et al. Revascularization versus medical therapy for renalartery stenosis. N Engl J Med. 2009;361:1953-62. doi: 10.1056/NEJMoa0905368.

3. Jaff MR, Bates M, Sullivan T, Popma J, Gao X, Zaugg M, et al. Significant reduction in systolic blood pressure following renal artery stenting in patients with uncontrolled hypertension: results from the HERCULES trial. Catheter Cardiovasc Interv. 2012;80:343-50. doi: 10.1002/ccd.24449.

4. Cooper CJ, Murphy TP, Cutlip DE, Jamerson K, Henrich W, Reid DM, et al; CORAL Investigators. Stenting and medical therapy for atherosclerotic renal-artery stenosis. N Engl J Med. 2014;370:13-22. doi: 10.1056/NEJMoa1310753.

5. Onuigbo MA. Stenting may benefit select patients with severe renal artery stenosis. Cleve Clin J Med. 2015;82:4957. doi: 10.3949/ccjm.82a.14128.

6. Ascer E, Gennaro M, Rogers D. Unilateral renal artery revascularization can salvage renal function and terminate dialysis in selected patients with uremia. J Vasc Surg. 1993;18:1012-8.

7. Gill IS, Novick AC, Hodge EE. Extra-anatomic renal revascularization in patients with renal artery stenosis and abdominal aortic occlusion. Urology. 1993;42:630-4.

8. Fergany A, Kolettis P, Novick AC. The contemporary role of extra-anatomical surgical renal revascularization in patients with atherosclerotic renal artery disease. J Urol. 1995;153:1798-801.

9. Sahay M, Saivani Y, Vali PS, Ismal K, Madhusudan. Splenorenal graft: A safe and effective treatment for renovascular hypertension. Indian J Nephrol. 2015;25:3702. doi: 10.4103/0971-4065.157417.

10. Somalanka S, Harris FE, Chemla E, Suckling RJ, Swift PA. Spleno-renal artery transposition in a solitary functioning kidney for treatment-resistant hypertension and acute kidney injury. BMJ Case Rep. 2017;2017:bcr-2017-220438. doi: 10.1136/bcr-2017-220438.

11. Bakris GL, Weir MR. Angiotensin-converting enzyme inhibitor-associated elevations in serum creatinine: is this a cause for concern? Arch Intern Med. 2000;160:685-93.

Copyright (C) 2019 The Author(s); Published by Nickan Research Institute. This is an open-access article distributed under the terms of the Creative Commons Attribution License (http://creativecommons.org/licenses/by/4.0), which permits unrestricted use, distribution, and reproduction in any medium, provided the original work is properly cited. 